to occur in postmenopausal women during pioglitazone treatment. Changes in marker levels indicated that the mechanism of BMD decline involved alterations to bone composition rather than bone turnover.

Glintborg and colleagues suggest that BMD probably declines throughout pioglitazone treatment, even in women with PCOS.

Original article Glintborg D et al. (2008) Association of pioglitazone treatment with decreased bone mineral density in obese premenopausal patients with polycystic ovary syndrome: a randomized, placebo-controlled trial. J Clin Endocrinol Metab 93: 1696-1701

\section{Identification of a serum biomarker profile that could aid early diagnosis of osteoporosis}

The key to the early diagnosis of osteoporosis is the measurement of BMD, augmented by accurate measurements of bone turnover. However, clinically assessed biochemical markers of bone turnover lack sensitivity and specificity, which prompted Bhattacharyya and colleagues to use surface-enhanced laser desorption ionization (SELDI) time-of-flight mass spectrometry (TOF-MS) to search for serum biomarker patterns that reflect bone turnover.

The researchers analyzed sera from postmenopausal women whose urinary levels of crosslinked $\mathrm{N}$-telopeptides of type I collagen indicated low or normal bone turnover $(n=28)$ or high bone turnover $(n=30)$. SELDI-TOF-MS and data analysis identified a specific pattern of protein peaks that discriminated between these groups; thereafter, SELDI-TOF-MS analysis of an independent dataset (sera from 59 different postmenopausal women, obtained 2 years after those from the first group to confirm the technique's reproducibility) successfully validated the diagnostic accuracy of this serum biomarker profile. Furthermore, individual protein peaks were significantly correlated with BMD status. Four protein peaks were identified by sequencing as proteolytic fragments of a kallikrein-sensitive glycoprotein involved in the host response system; the authors hypothesize that the serum protein fingerprint identified reflects levels of osteoclast activity.

The authors conclude that their serum biomarker profile accurately distinguished women with high bone turnover from those with low or normal bone turnover, and correlated with the individual's BMD status; they urge further study to confirm these findings.

Original article Bhattacharyya S et al. (2008) A serum biomarker profile associated with high bone turnover and bone mineral density in postmenopausal women. J Bone Miner Res [doi:10.1359/jbmr.080235]

\section{Patients with MEN1-associated ZES have a severe form of hyperparathyroidism}

The most common clinical manifestation of multiple endocrine neoplasia type 1 (MEN1) is primary hyperparathyroidism, which is usually treated by parathyroidectomy. Zollinger-Ellison syndrome (ZES) is the most common functional neuroendocrine syndrome associated with MEN1. Nonetheless, few studies have focused on long-term outcomes after parathyroid surgery in this subset of patients.

Norton et al. studied 84 patients with MEN1, hyperparathyroidism and ZES, who underwent parathyroid surgery and were subsequently assessed at intervals of 1-3 years (mean follow-up 7.2 years).

Among the 35 patients in whom $>3$ parathyroid glands were removed, the incidence of hypoparathyroidism was 3\% and that of persistent or recurrent hyperparathyroidism was $92 \%$. Among the 40 patients in whom 3-3.5 glands were resected, hypoparathyroidism occurred in 10\% and persistent or recurrent hyperparathyroidism in $54 \%$. Hypothyroidism occurred in $22 \%$ and recurrent hyperparathyroidism in $55 \%$ of patients after 4-gland resection. The disease-free interval was significantly prolonged if 3.5-4 glands were removed. Successful surgical treatment of hyperparathyroidism also significantly improved biochemical parameters of ZES.

The authors conclude that individuals with MEN1, hyperparathyroidism and ZES have a particularly severe form of parathyroid hyperplasia and require careful long-term follow-up. The authors recommend parathyroidectomy with a 3.5-gland resection for such patientssuch surgery is associated with a prolonged disease-free interval, an acceptable incidence of hypoparathyroidism and a favorable effect on biochemical parameters of ZES.

Original article Norton JA et al. (2008) Prospective study of surgery for primary hyperparathyroidism (HPT) in multiple endocrine neoplasia-type 1 and Zollinger-Ellison syndrome: long-term outcome of a more virulent form of HPT. Ann Surg 247: $501-510$ 